\title{
Repair of impaired myocardium by means of implantation of engineered autologous myoblast sheets
}

\author{
Imran A. Memon, MBBS, PhD, ${ }^{a}$ Yoshiki Sawa, MD, PhD, ${ }^{a}$ Norihide Fukushima, MD, a Goro Matsumiya, MD, ${ }^{a}$ \\ Shigeru Miyagawa, MD, PhD, ${ }^{a}$ Satoshi Taketani, MD, ${ }^{a}$ Satoru K. Sakakida, MD, PhD, ${ }^{\mathrm{c}}$ Haruhiko Kondoh, MD, PhD, \\ Alexey N. Aleshin, MD, PhD, ${ }^{a}$ Tatsuya Shimizu, MD, PhD, ${ }^{\mathrm{b}}$ Teruo Okano, PhD, ${ }^{\mathrm{b}}$ and Hikaru Matsuda, MD
}

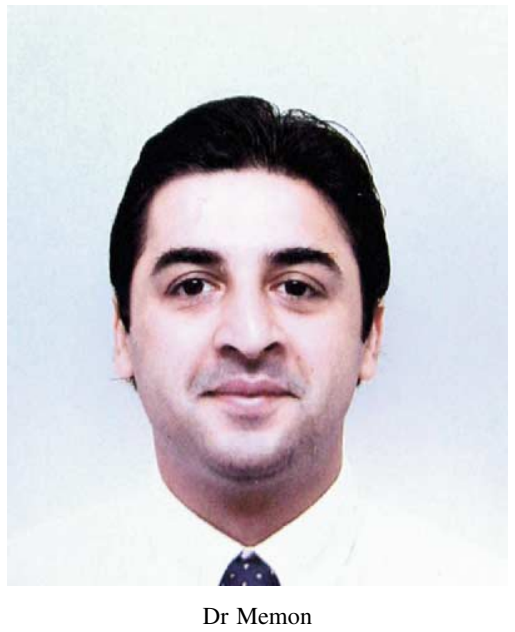

From the Division of Cardiovascular Surgery, Department of Surgery, ${ }^{\mathrm{a}}$ and Molecular Pharmacology, ${ }^{\mathrm{c}}$ Osaka University Graduate School of Medicine, ${ }^{\text {a }}$ Osaka, Japan, and Tokyo Women's Medical University, Institute of Advanced Biomedical Engineering and Science, ${ }^{\mathrm{b}}$ Tokyo, Japan.

Received for publication March 10, 2005; revisions received July 17, 2005; accepted for publication July 19, 2005

Address for reprints: Yoshiki Sawa, MD, Department of Surgery E1, Division of Cardiovascular Surgery, University Graduate School of Medicine, 2-2 Yamada-Oka, Suita, Osaka 565-0871, Japan (E-mail: i.memon@ victorchang.unsw.edu.au).

J Thorac Cardiovasc Surg 2005;130:1333-41 $0022-5223 / \$ 30.00$

Copyright () 2005 by The American Association for Thoracic Surgery

doi:10.1016/j.jtcvs.2005.07.023
Objectives: Autologous skeletal myoblast cell transplantation by means of the injection method is subject to the loss of intercellular communication, extracellular matrix, and cell numbers. We hypothesize that the implantation of skeletal myoblast cell sheets might be more advantageous in repairing the impaired heart by providing uniform and stable cell delivery with less cell loss and without disrupting the cell-cell microenvironment.

Methods: Left anterior descending coronary artery-ligated Lewis rat hearts (2 weeks, total $\mathrm{n}=173$ ) received $1 \times 10^{7}$ autologous skeletal myoblasts by means of cell transplantation either through myoblast injection or implantation of 2 monolayerconstructed myoblast sheets $\left(5 \times 10^{6}\right.$ cells per sheet $)$ or through medium injection. Myoblast sheets were constructed with temperature-responsive, polymer-grafted cell-culture dishes, which release the confluent cells from the dish surface at less than $20^{\circ} \mathrm{C}$.

Results: Echocardiographic results indicated higher improvement of cardiac performance in the myoblast sheet group than among the other groups until 8 weeks after cell transplantation. Histologic comparison revealed greater cellularity and abundant widespread neocapillaries within the noticeable uniform thickened wall in myoblast sheet group hearts only. Fibrosis was substantially reduced with skeletal myoblast sheet implantation compared with skeletal myoblast cell injection. Obviously higher numbers of hematopoietic stem cells (c-kit, stem cell antigen 1, and CD34) were observed in the myoblast sheet group infarct heart region. Reverse transcriptionpolymerase chain reaction results showed expression of stromal-derived factor 1 , hepatocyte growth factor, and vascular endothelial growth factor as follows: myoblast sheets $>$ myoblast injection $>$ control.

Conclusions: Myoblast sheets repaired the impaired myocardium, reduced fibrosis, and prevented remodeling in association with recruitment of hematopoietic stem cells through the release of stromal-derived factor 1 and other growth factors. Our experiment indicates a therapy for patients with severe heart failure.

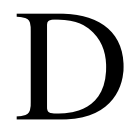
evastating myocardial infarction leads to heart failure if left untreated and unsupported. After damage, the heart is capable of a very limited regenerative capacity. ${ }^{1,2}$ Heart transplantations and mechanical support with left ventricular (LV) assist systems are supportive in patients with heart failure but are subject to a few important limitations, such as organ rejection, donor shortage, infections, and durability of the assist systems. Autologous cellular cardiomyoplasty (cell transplantation [Ctx]) overcomes these shortcomings and has emerged as an independent potential therapy to regenerate-repair lost myocardium and improve cardiac function. ${ }^{3}$ 


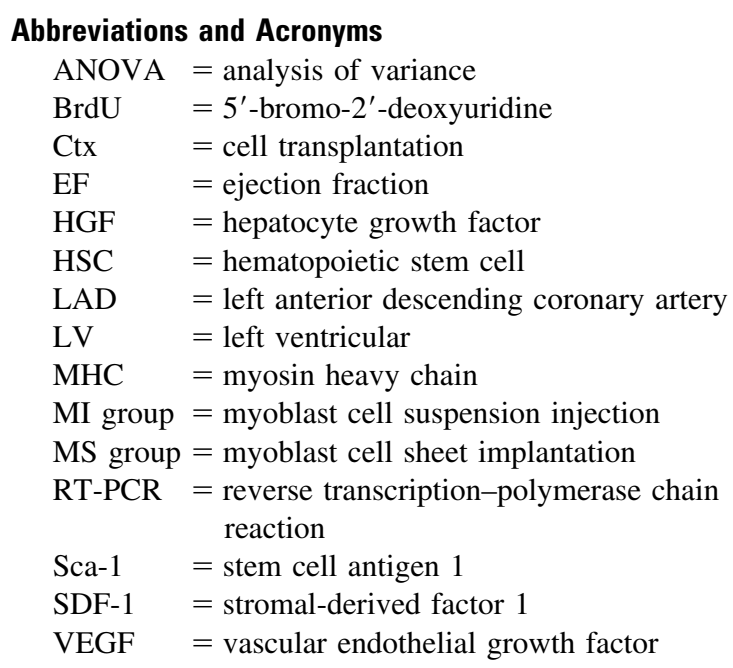

Skeletal myoblasts maintain muscle regenerative quality with growing power to millions and act as the most suitable autologous cell type for clinical application. ${ }^{4}$ Several laboratories have reported improved heart performance with myoblast transplantation. ${ }^{5-7}$ However, the use of trypsin to detach the cells from the culture dish disrupts their microintercellular communication and extracellular matrix, restricts cell survival and growth, and thus appears deleterious to Ctx therapy. ${ }^{8}$ Intercellular communication factors play a key role in cell adhesion, migration, proliferation, differentiation, and death ${ }^{9}$ and must be maintained for optimal cellular benefits. We believe that the selection of a reliable cell delivery mode that maintains the potentiality of the cells is as valuable as the type and the number of cells.

Tissue engineering for tissue regeneration fulfills the criteria to repair the heart and eliminates several drawbacks. ${ }^{10}$ The use of scaffold for tissue engineering is supportive for myocardial regeneration but subject to biocompatibility, biodegradability, and cytotoxicity, including inflammatory response and surface adhesion molecule loss issues, and this limits its efficacy. Eliminating such disadvantages, we have established cell sheet engineering technology without using scaffolds. ${ }^{11}$ The engineered cell sheets from this technique showed preserved cellular communica- tion junctions, endogenous extracellular matrix, and integrative adhesive agents. ${ }^{12}$ Nonligature implantation of our engineered neonatal cardiomyocyte sheets to infarcted myocardium showed their integration with impaired myocardium and improved cardiac performance. ${ }^{13}$ For clinical application, use of skeletal myoblasts averts ethical and cell source issues. Thus we selected autologous skeletal myoblasts and constructed myoblast sheets.

Recent findings suggested that locally or transgenically delivered stromal-derived factor 1 (SDF-1) expression plays a role in mobilizing and recruiting stem cells with neovascularization. ${ }^{14}$ Because SDF-1 is secreted in skeletal muscle tissue, ${ }^{15}$ grafted myoblasts might beneficially attract hematopoietic stem cells (HSCs) to home in the infarct heart area for heart regeneration and angiogenesis.

We proceeded to investigate whether the myoblast sheets serve as a suitable and potential cell carrier and cell deliverer for heart repair, attract hematopoietic stem cells-progenitor cells by releasing SDF-1, and tame cardiac remodeling to improve deteriorated heart geometry and function.

\section{Materials and Methods \\ Animals}

One hundred seventy-three female and male Wistar-Lewis rats (180-220 g, 6-10 weeks old; Seac Yoshitomi Ltd) were used for myoblast donors and recipients for this study. Humane animal care was used in compliance with the "Principles of Laboratory Animal Care" formulated by the National Society for Medical Research and the "Guide for the Care and Use of Laboratory Animals" prepared by the Institute of Animal Resources and published by the National Institutes of Health (National Institutes of Health publication no. 86-23, revised 1985). Distribution of 173 rats is shown in Table 1.

\section{Isolation of Myoblasts and Construction of Myoblast \\ Sheets}

Isolation and expansion of myoblasts was performed $(n=40$ rats in the myoblast cell suspension injection [MI] group and $n=43$ rats in the myoblast cell sheet implantation [MS] group and each Ctx group) with a modified protocol, as previously described. ${ }^{16}$ Cells were delivered to the infarcted hearts either by means of direct intramyocardial injection or implantation of constructed myoblast sheets.

Custom-made culture dishes coated with thermoresponsive polymers and poly- $N$-isopropylacrylamide were used to construct

TABLE 1. Distribution of animals

\begin{tabular}{lccccc}
\hline Groups & $\begin{array}{c}\text { Cell therapy } \\
\text { animals (total) }\end{array}$ & $\begin{array}{c}\text { Echocardiography at 2, } \\
\text { 4, and 8 weeks }\end{array}$ & $\begin{array}{c}\text { Staining at } \\
\mathbf{1 4} \mathbf{d}^{*}\end{array}$ & $\begin{array}{c}\text { RT-PCR at 7 d/DB at 7 } \\
\text { and 14 d }\end{array}$ & $\begin{array}{c}\text { Animal no.: } \\
\text { myoblast isolation }\end{array}$ \\
\hline MS & $29+1 \dagger$ & $\mathrm{n}=5$ & $\mathrm{n}=10$ & $\mathrm{n}=14$ & $\mathrm{n}=43$ \\
MI & $25+5 \dagger$ & $\mathrm{n}=5$ & $\mathrm{n}=10$ & $\mathrm{n}=10$ & $\mathrm{n}=40$ \\
$\mathrm{C}$ & $29+1 \dagger$ & $\mathrm{n}=5$ & $\mathrm{n}=9$ & $\mathrm{n}=15$ & $\mathrm{n}=0$ \\
\hline
\end{tabular}

Total = 173 Lewis rats. $R T-P C R$, Real-time reverse transcription-polymerase chain reaction; $D B$, double immunofluorescence staining; $M S$, myoblast sheet; $M I$, myoblast injection; $C$, medium injection. *Histologic staining (hematoxylin and eosin, Masson trichrome, factor VIII). $†$ Mortality number. 

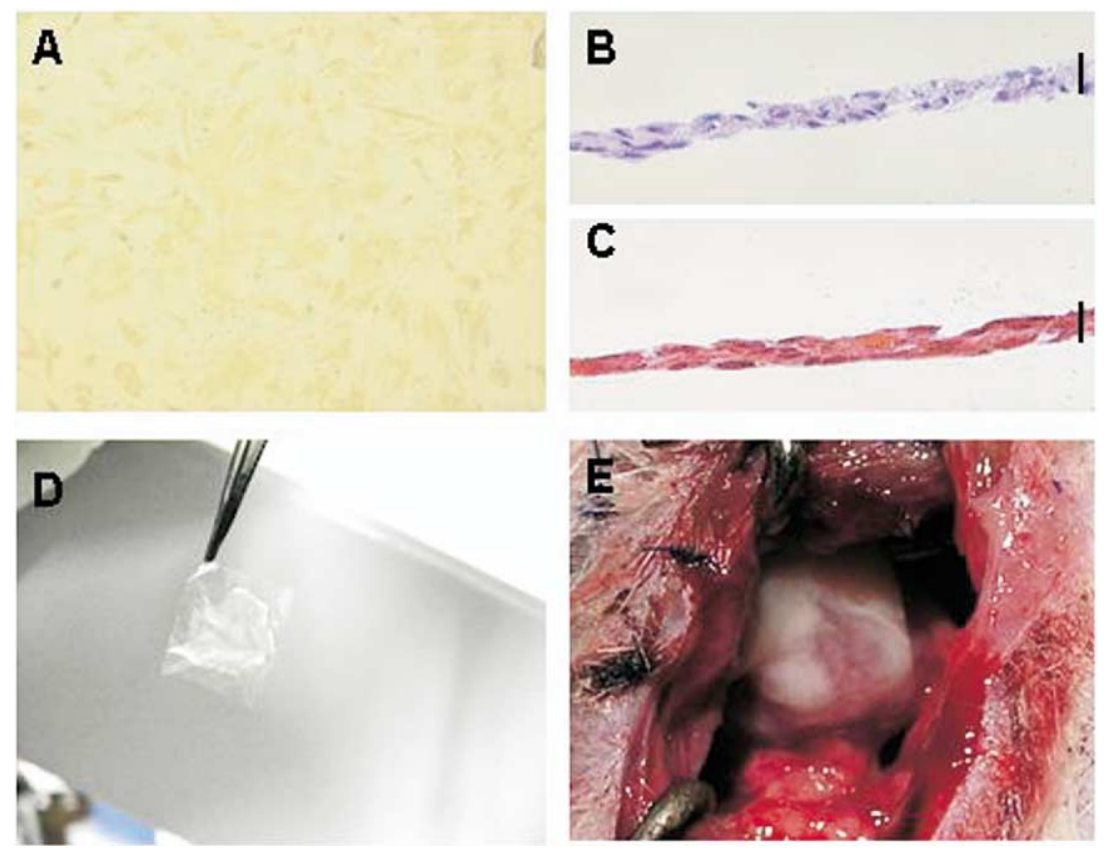

Figure 1. Immunohistochemical staining ( $A$; $40 \times$ magnification) showed that roughly $60 \%$ to $70 \%$ of cultured cells were desmin-positive muscle lineage cells. Hematoxylin and eosin (B) and Masson trichrome staining (C; 400X magnification) indicated high cellularity and low fibrous content of the myoblast sheet (scale bar $=50 \mu \mathrm{m}$ ). Two sheets were piled to make a composite sheet graft (D). Grafts implanted to the infarcted area of a rat heart are shown in $E$. the myoblast sheets, as previously described. ${ }^{17}$ The surfaces of these dishes are hydrophobic at $37^{\circ} \mathrm{C}$ and become hydrophilic at temperatures of less than $20^{\circ} \mathrm{C}$. The cell suspensions with a density of $5 \times 10^{6}$ were plated in the thermoresponsive cell-culture dish at $37^{\circ} \mathrm{C}$ in an incubator for 1 to 2 days. The confluent cells cultured on these dishes at $37^{\circ} \mathrm{C}$ are spontaneously detached by placing them at $20^{\circ} \mathrm{C}$ for 1 hour. Free monolayer cell sheets are thus attained in the manner. The size of these cell sheets ranged to $1.00 \pm$ $0.05 \mathrm{~cm}^{2}$ in area and $50 \pm 10.0 \mu \mathrm{m}$ in thickness (Figure $1, B$ and $C)$. Finally, 2 sheets were piled to make a thicker sheet for grafting. Thus one graft for implantation contained around $1.0 \times 10^{7}$ cells. Cell count from a single myoblast sheet showed recovery of $5 \times$ $10^{6} \pm 5693.96$ cells.

\section{Surgical Procedure}

The anesthetized rats (ketamine and xylazine) were intubated, and positive-pressure ventilation was maintained with a ventilator (model SN-480-7 Shinano). Thoracotomy was performed between the fourth and fifth intercostal spaces, and acute myocardial infarction was induced in the rats by means of ligation of the left anterior descending coronary artery (LAD), as previously described. ${ }^{18}$ After 2 weeks, depending on the mode of therapy, the rats were randomly divided into 3 treatment groups: (1) MS group $\left(\mathrm{n}=29,1 \times 10^{7}\right.$ cells per sheet $) ;(2)$ MI group $\left(\mathrm{n}=25,1 \times 10^{7}\right.$ cells in $0.2 \mathrm{~mL}$ of Dulbecco's modified Eagle's medium); and (3) medium injection group (control group, $\mathrm{n}=29$ ). The cells in the MI group were transplanted by injecting at 5 sites in and around the infarcted region with a 30-gauge tuberculin syringe, and the same number of cells was delivered in the MS group by implanting the cell sheets directly over the scar area without sutures. After treatment, a biodegradable collagen sheet $(\varnothing 33 \mathrm{~mm}$; Kokencellgen) was overlapped on the hearts to avoid adhesion to the chest wall (Figure 1,E).

\section{Assessment of Cardiac Performance}

After achievement of anesthesia, heart function was monitored at baseline ( 2 weeks after LAD ligation and before Ctx) and at 2, 4, and 8 weeks after $\mathrm{Ctx}(\mathrm{n}=5$ in each group at each time point). Echocardiography was performed with an ultrasound machine (SONOS 5500, Agilent Technologies) equipped with an annular array transducer operating at $12 \mathrm{Mhz}$. The heart was first imaged in the 2-dimensional mode in short-axis views at the level of the largest LV diameter. The systolic and diastolic LV area was determined at the same time. The calculation of LV volume was based on the LV short-axis area. This view was used to position the M-mode cursor perpendicular to the LV anterior and posterior wall. Diastolic measurements were measured at the time of the apparent maximal LV diastolic dimension. The LV end-systolic dimension was measured at the time of the most anterior systolic excursion of the LV posterior wall. The LV dimension at end diastole and LV dimension at end systole were determined. Minor axis anterior-inferior wall dimensions were used. Dimensions and area data are presented as the average of measurements of 3 selected beats. LV ejection fraction (EF) was calculated by using the following formula: $\operatorname{LVEF}(\%)=\left[\left(L V D d^{3}-\right.\right.$ $\left.\left.L V D s^{3}\right) / L V D d^{3}\right] \times 100$. End-diastolic area and end-systolic area were determined to evaluate the percentage of fractional area shortening by using the following formula: $F A S(\%)=E D A-$ $E S A / E D A \times 100(\%)$.

\section{Histologic Examination}

Hematoxylin and eosin and Masson trichrome staining were carried out 2 weeks after the treatments to evaluate the histologic nature of infarcted lesions in each group. Treated hearts from each group (MS group, $\mathrm{n}=10$; MI group, $\mathrm{n}=10$; control group, $\mathrm{n}=$ 9) were removed and fixed with $10 \%$ formalin. At least 3 step 
sections at the middle portion of the left ventricle were prepared for each heart, and anterior wall thickness was measured in the sections.

Paraffin blocks were sectioned, deparaffinized, and immunostained with the following antibodies: monoclonal anti-skeletal myosin antibody to fast-twitch skeletal myosin heavy chain (MHC; MY-32, Sigma, St Louis, Mo) and monoclonal anti-myosin (skeletal, slow) directed against skeletal slow MHC isoforms (NOQ7.5.4D, Sigma).

Immunohistochemical staining for von Willebrand factor antigen was performed with DAKO's EPOS von Willebrand factor/ horseradish peroxidase kit (DakoCytomation, Denmark) to detect vessels in the infarcted area, according to the manufacturer's protocol. Ten low-power fields per section were analyzed to assess the vascular density at $100 \times$ magnification. The results were expressed as the number of blood vessels per square millimeter.

The percentage of the total fibrotic area was quantified by means of image analysis of the Masson trichrome-stained sections by means of the planimetric method with MetaMorph software (UIC). Ten low-power fields per section were analyzed to assess the fibrous contents in the infarcted area (Olympus, Japan).

\section{Characterization of Dividing Cells on the Basis of 5'-Bromo-2' -Deoxyuridine Administration}

$5^{\prime}$-Bromo-2'-deoxyuridine (BrdU), $50 \mathrm{mg} \cdot \mathrm{kg}^{-1} \cdot \mathrm{d}^{-1}$, was intraperitoneally injected into the treated rats for 5 consecutive days beginning from the day of myoblast implantation, as previously described, to identify and characterize dividing cells in infarcted areas of the treated hearts. ${ }^{14}$ The numbers of animals for each treatment group in this set of experiments was 14 for the MS group, 10 for the MI group, and 15 for the control group. The hearts were removed 7 or 14 days after the implantation of myoblasts and cut into 2 pieces. One set of pieces was frozen in liquid nitrogen and sectioned for immunohistochemical staining. The other half was stored in RNAlater solution (QIAGEN) for subsequent RNA extraction.

The anti-BrdU In-Situ Detection Kit (BD Pharmagen) was used to observe the BrdU-positive cells in the infarcted heart, according to the manufacturer's protocol. The sections of experimental cardiac muscle tissue were immunostained with antibodies against CD34, c-kit (CD117), von Willebrand factor (all from Santa Cruz, Inc), stem cell antigen 1 (Sca-1; BD Inc), and Troponin-T (NeoMarkers). For immunostaining, tissue was fixed for 10 minutes with $4 \%$ paraformaldehyde. After the primary antibody incubation, the following secondary antibodies (all material from Molecular Probes, Inc) were used: goat anti-mouse Alexa Flour 488, goat anti-mouse Alexa Flour 546, goat anti-rabbit Alexa Flour 546, and donkey anti-goat Alexa Flour 546. After the double primary antibody staining, BrdU staining was performed (anti-bromodeoxyuridine, mouse $\mathrm{IgG}_{1}$, monoclonal PRB-1, Alexa Fluor 488 conjugate). Cover slips were finally mounted with SlowFade Antifade kit reagents with 4,6-diamino-2-phenylindole nuclear dye. Slides were viewed with a fluorescent microscope equipped with differential interference contrast optics (Polaroid, Olympus, Japan). The number of stained cells per square millimeter at 10 different fields $(100 \times)$ was counted.

\section{Quantitation of Gene Transcripts by Means of Real-Time Reverse Transcription-Polymerase Chain Reaction}

Total RNA was isolated from the stored specimen with RNeasy Mini Kit (QIAGEN) and reverse transcribed with Omniscript Reverse Transcriptase (QIAGEN). Real-time reverse transcription-polymerase chain reaction (RT-PCR) was performed with ABI PRISM 7700, as previously described. ${ }^{19,20}$ The sequence of forward primers, reverse primers, and probes for SDF- 1 and reduced glyceraldehyde-phosphate dehydrogenase is not shown. The measurement for each gene, each RNA sample, was performed in triplicate. RT-PCR was performed to measure the expression of SDF-1, hepatocyte growth factor (HGF), and vascular endothelial growth factor (VEGF) in only specimens stored for 7 days.

\section{Statistical Analysis}

All data are presented as means \pm standard deviation and subjected to multiple analysis of variance (ANOVA) with StatView 5.0 software (Abacus Concepts). Time-course data shown in Figure 2 were first analyzed by using repeated-measurements 2-way ANOVA, and further comparisons for the specific 2 groups were carried out with the Tukey-Kramer post hoc test. The other numeric data were analyzed by using 1-way ANOVA, and the significance of the comparisons was tested with the Bonferroni method.

\section{Results}

\section{Effect of Myoblast Transplantation on Cardiac Performance}

Myocardial infarction resulted in less than $20 \%$ acute mortality within 24 hours of the operation (not added to total number of animals). Few animals died at the time of reoperation (MS group, $\mathrm{n}=1$; MI group, $\mathrm{n}=5$; and control group, $n=1)$. Ventricular remodeling after weeks of LAD ligation characteristically resulted in both global cavity enlargement and cardiac pump dysfunction, as measured with echocardiography at baseline. At 2, 4, and 8 weeks, control group hearts showed deterioration in EF, with dilatation of the ventricular chamber. The MI group showed improved EF (2, 4, and 8 weeks) compared with that seen in the control group but did not show any significant reduction in LV chamber dilatation compared with the other groups. However, sheet implants in the MS group resulted in significant improvement in EF at 2, 4, and 8 weeks compared with that seen in the control group and significant improvement in EF at 2 and 4 weeks compared with that seen in the MI group. The reduction in LV chamber area was evident after treatment in the MS group only. LV end-systolic area was significantly reduced in the MS group at 4 and 8 weeks, whereas LV end-diastolic area was significantly reduced in the MS group at 4 and 8 weeks. The percentage of fractional area shortening was significantly improved in the MS group compared with in the other groups at 2 and 4 weeks (Figure 2). 

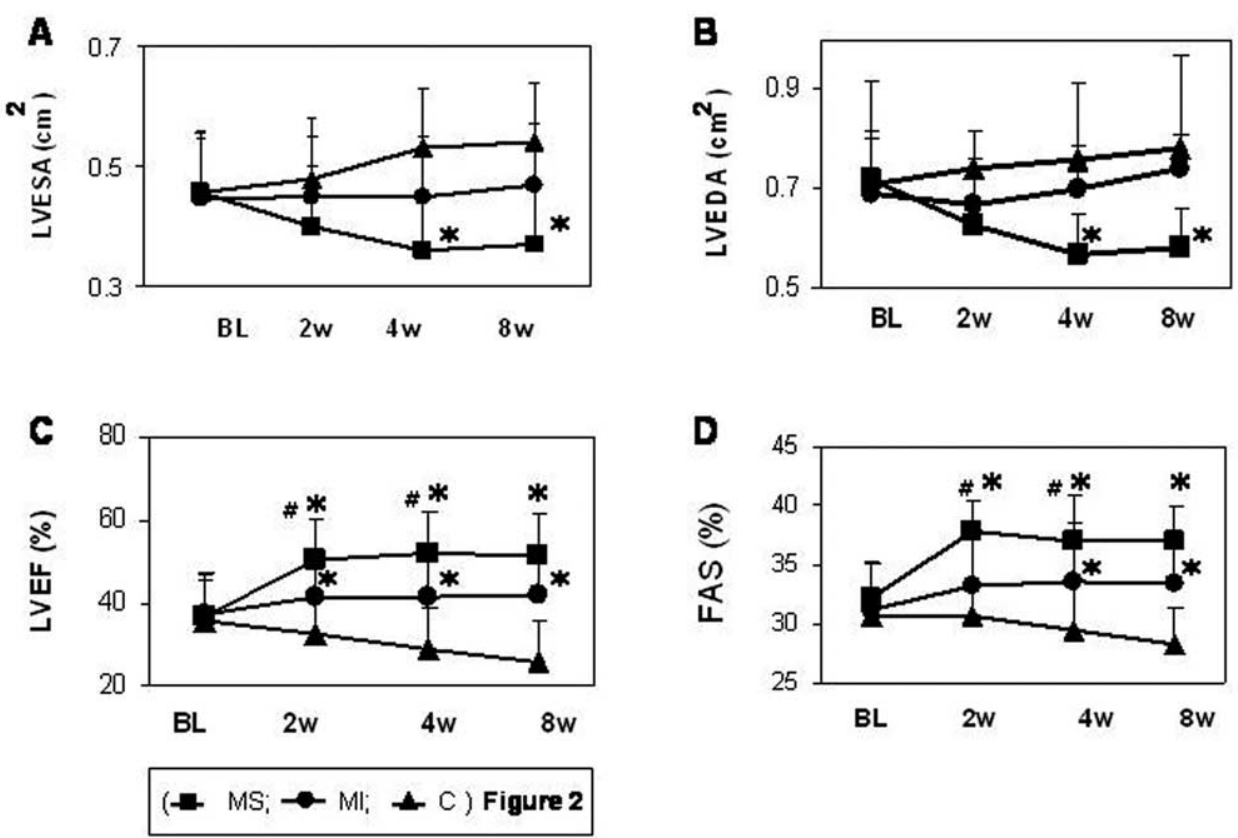

Figure 2. Improved cardiac performance was observed in myoblast sheet implantation group hearts. Baseline represents 2 weeks after the left anterior descending coronary artery ligation. $A$, left ventricular end-systolic area (LVESA); B, left ventricular end-diastolic area (LVEDA); C, left ventricular ejection fraction (LVEF); D, Percentage fractional area shortening (FAS). ${ }^{*} P<.05$ versus control group; $\#<.05$, myoblast sheet group versus myoblast injection group. $B L$, Baseline; $M S$, myoblast sheet group; $M I$, myoblast injection group; $C$, control group.

\section{Histologic Findings}

Before myoblast implantation, the anterior wall of the left ventricle was already replaced by fibrous scar, and it was much thinner compared with other healthy parts of the left ventricle (Figure 3, baseline). Two weeks after the treatment, the control and MI groups showed similar morphology to that observed at baseline, although the MI group showed isolated cells inside the scar tissues. However, MS group hearts revealed that the anterior wall was richly cellularized and mainly occupied by round or oval-shaped cellular contents, which were uniformly distributed within the anterior wall, replacing the scar area (Figure 3, A). The MS group showed a uniform and significantly thicker anterior wall (Figure 3, B). There was no major difference in the thickness of the anterior walls among the baseline, MI, and control groups. Significant reduction of myocardial fibrosis was observed in the anterior wall of the hearts in the MI group when compared with that seen in the control group. However, the reduction of fibrosis was much more evident in the MS group (Figure 3,C) compared with that seen in the MI and control groups. Histologic observations and quantifications were mainly conducted 14 days after Ctx.

A cellular layer (partial or complete) of elongated myofibers was observed in the epicardial region in MS group hearts. In MI group hearts we observed small and isolated myofibers, mainly in the midmyocardial region. After 2 week of Ctx, serial sections immunostained by directing against the fast $\mathrm{MHC}$ isoform indicated the graft occupation by skeletal muscle structures, mainly fast-twitch fibers. A small percentage, approximately $20 \%$, stained positive for slow MHC, showing conversion to slow-twitch myofibers. In the MI group we observed small and isolated myofibers surrounded by collagenous contents, mainly in the midmyocardial region.

Local capillary density in the anterior wall was evaluated in each group. Both the MI and MS groups showed increases in the number of local capillaries. Quantification of the vascular density at 2 weeks showed obviously higher numbers of vessels in the MS group $\left(26.1 \pm 4.7 \times 10^{2}\right.$ / $\left.\mathrm{mm}^{2}, P<.0001\right)$ versus the MI group $(14.4 \pm 3.4 \times$ $\left.10^{2} / \mathrm{mm}^{2}\right)$ and versus the control group $\left(4.1 \pm 2.1 \times 10^{2} /\right.$ $\left.\mathrm{mm}^{2}\right)$. The MI group $\left(14.4 \pm 3.4 \times 10^{2} / \mathrm{mm}^{2}\right)$ showed significantly higher vascular density versus the control group $\left(4.1 \pm 2.1 \times 10^{2} / \mathrm{mm}^{2}, P<.0001\right)$.

\section{Immunofluorescence Staining}

Double staining count for BrdU and different cells markers (c-kit, Sca-1, and CD34) at 14 days showed a greater presence of these cells in MS hearts compared with in MI and control hearts (Figure 4, A). 

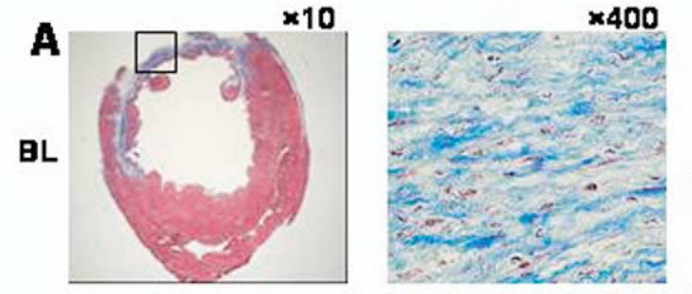

\section{B}

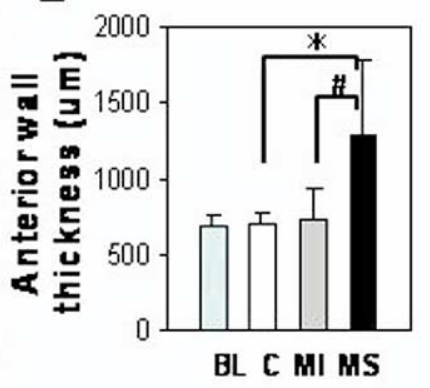

c
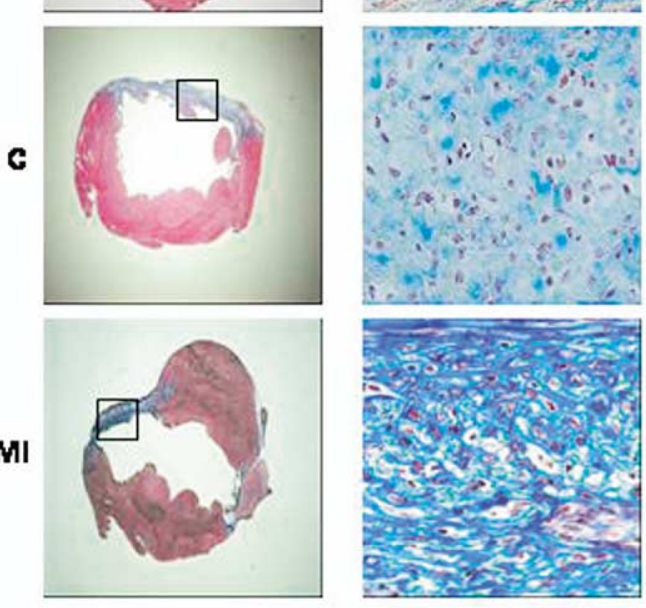

MS

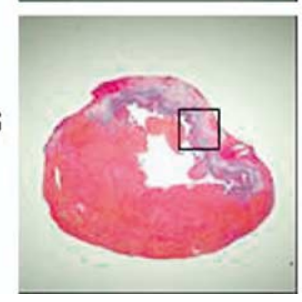

Figure 3. A, Macroscopic $(10 \times)$ and microscopic $(400 \times)$ view of hearts from baseline $(B L)$, control $(C)$, myoblast injection (MI), and myoblast sheet implantation (MS) groups are shown. The baseline hearts that were excised 2 weeks after left anterior descending coronary artery ligation showed complete scar formation in the infarcted area, and the area in the control group showed similar findings. Histology was also similar in the MI group, although we observed dispersed cellularity in the scar area. The infarcted areas in the MS group were occupied with highly cellular tissues. The anterior wall was significantly thicker in the MS group only compared with the MI and control groups (B). The degree of myocardial fibrosis was similar in the baseline and control groups, and it was reduced in the MI group (C). The percentage of fibrosis in the MS group, however, was markedly less compared with that in the MI and control group. ${ }^{*} \boldsymbol{P}<.05$ versus control group; \#P<.05, MS group versus MI group.
Fourteen days after Ctx, the number of c-kit, Sca-1, and CD34 cells in the infarct heart zone of animals treated with myoblast sheets was significantly higher per square millimeter compared with that seen in the animals treated with myoblast injection and the control group (Figure 4,C). At day 7 , the number of these positively stained cells was slightly higher in all groups compared with on day 14 but significantly higher in the MS group only (data not shown).

\section{Assessment of Growth Factors by Gene Expression} RT-PCR analysis after 1 week of Ctx showed higher expression of SDF-1 in the MS group compared with that in the control groups as follows: MS group $(0.09 \pm 0.02, P<$ $.05)>$ MI group $(0.05 \pm 0.02)>$ control group $(0.02 \pm$ 0.01 ; Figure 4, $D$ ). Expression of the genes encoding SDF-1 and HGF was lowest in the infarcted area of control group hearts, and they tended to increase in those of the MI group $(P<.05)$. RT-PCR results also showed significantly higher expression of growth factors (HGF and VEGF) by MS group hearts after the myoblast sheet implantation compared with after Ctx by means of needle injection in MI group hearts and control group hearts (Figure 4, E and $F$ ), whereas expression levels of VEGF in the control and MI groups were similar.

\section{Discussion}

In this study we aimed to determine whether engineered autologous myoblast sheets repair the infarcted myocardial wall to attenuate cardiac remodeling for improving cardiac performance.

The concept of regenerative medicine and tissue engineering is to deliver healthy cells to the damaged or diseased tissues of the organ and stimulate their endogenous system of repair and regeneration for the desired organs. ${ }^{21}$ Tissue engineering, which is based on scaffold use, rescues the damaged tissue through tissue regeneration. However, Zimmermann and colleagues ${ }^{22}$ and Kofidis and associates ${ }^{23}$ have reported several potential drawbacks accompanying scaffold tissue constructs.

Beneficial for Ctx, as reported by Kushida and coworkers, ${ }^{24}$ the cell sheet tissue maintains intact membrane proteins and adhesive proteins, incorporates extracellular matrix molecules, and secretes growth factors caused by 

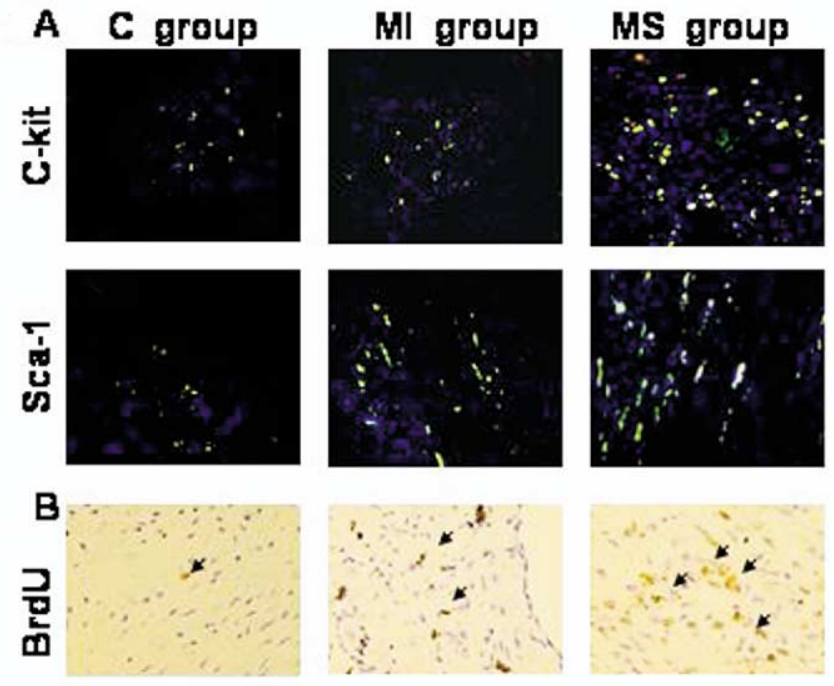
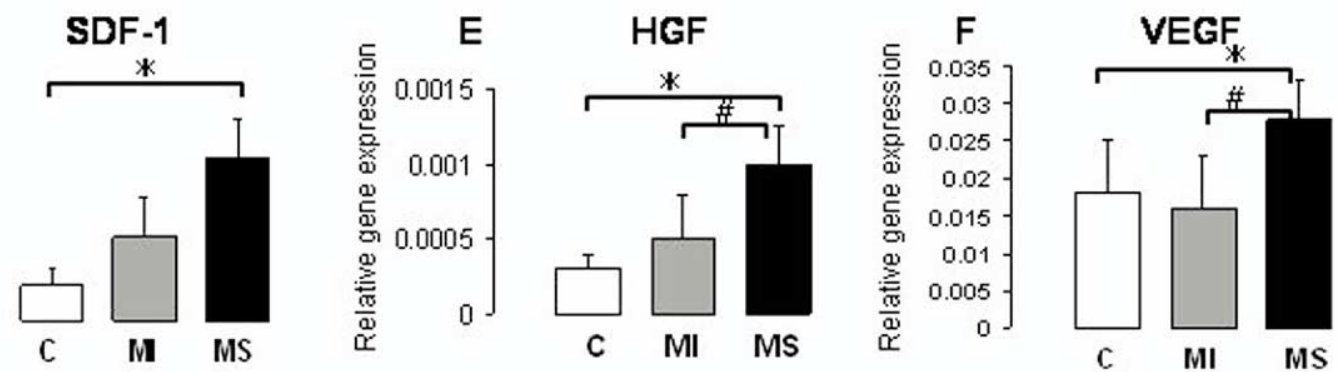

Figure 4. Greater cellularity was observed in the myoblast sheet (MS) group (A, right), whereas less cellularity was seen in the myoblast injection (MI) group (A, middle) and the control (C) group (A, left). Double-staining fluorescent images were merged to reveal areas of costaining, as evidenced by yellowish-orange staining pattern (400 $\times$ magnification). BrdU-positive cells were observed in the infarcted heart region with the anti-BrdU In-Situ Detection Kit (B). Higher numbers of HSCs were observed in the infarcted repairing myocardium in the MS group only (C). ${ }^{*} P<.05$ versus control group; $\# P<.05$, MS group versus MI group. Relative levels of expression for stromal-derived factor 1 (SDF-1; D), hepatocyte growth factor (HGF; E), and vascular endothelial growth factor (VEGF; F) genes was measured 1 week after myoblast implantation and was measured as significantly higher in the MS group. ${ }^{*} P<.05$ versus control group; $\# P<.05$ MS group versus MI group.

favorable cell-to-cell cross-talk and prevents cellular microenvironment disruption caused by enzymatic reactions (trypsin or dispase) for cell detachment from cell-culture dishes.

After myoblast sheet implantation, the MS group demonstrated uniform repair in the anterior wall, which probably played a primary role in reremodeling the geometry of the LV chamber. The reasoning for this attenuation of remodeling appears to be the efficient delivery of myoblast in the form of sheets that overlapped the scar area and its borders in the form of a cellular bridge. A primary and well-maintained cellular microenvironment in myoblast sheets probably supports all necessary cellular activities (eg, release of growth factors). In the case of the MI group, transplanted cells settle in the cavities surrounded by the fibrous scar without any intercellular communication and begin their basic but delayed establishment of microenvironment, which proves fatal for their primary survival.

The greater cellularity might be, additionally, a result of the potential role of released SDF-1 and other growth factors by transplanted cells, which involves cell migration, adhesion, and proliferation. We detected the expression of SDF-1 by the myoblast sheets in vitro (data not shown). Because HSCs bears CXCR4 receptors and establish a coupling balance with stem cell homing factor-specific ligand, SDF-1, and maintain their homing within the bone marrow, the recruitment of HSCs through SDF-1 release from myoblast sheets might associate with its mechanism 
for greater cellularity and repair. ${ }^{14,15}$ Similarly, the greater release of HGF and VEGF in MS group hearts compared with in MI group hearts might also play an advantageous role for damaged hearts. Myoblasts act as the natural supplier of growth factors, such as HGF, and proved to be an angiogenic and antifibrotic factor by using it for their own growth and recruitment of other cells. ${ }^{25}$

Findings by Balsam and colleagues ${ }^{26}$ demonstrated the continuing hematopoietic fate of HSCs in ischemic myocardium. Two weeks after Ctx, we similarly observed abundant c-kit, Sca-1, and CD34 marker expressing cells. Murry and associates ${ }^{27}$ stated the nontransdifferentiation of HSCs into cardiac myocytes, but the findings by Orlic and coworkers ${ }^{28}$ showing the extensive conversion of $\mathrm{Lin}^{-} \mathrm{c}-\mathrm{kit}^{+}$bone marrow cells to myocytes remain controversial. The exact extent of benefits by HSCs for better cardiac performance remains skeptical, but we agree with Askari and colleagues ${ }^{14}$ in stating that the provision of increased cellularity and replacement of the scar area by the presence of HSCs is primarily responsible for reversing the remodeling by increasing the LV mass and decreasing LV dilatation. The replacement of fibrosis by greater cellularity might be the benefit of HSCs.

Recent groups have reported the existence of cardiac stem cells in the heart for tissue repair, although its potentiality appears limited. ${ }^{29,30}$ However, several other works have reported bone marrow as a real origin of these different immigrant cells. ${ }^{31}$ These cells might mobilize under the influence of chemotactic factors and migrate to the tissue undergoing repair, either from bone marrow or from within the heart. In our case a few colocalized endothelial and cardiac-type cells with BrdU-positive cells were also rarely observed. Further investigations are needed to show the exact origin of these HSCs and endothelial cells and their exact transdifferentiation fate.

We have not quantitatively assessed and compared the size of grafted myoblasts in both groups; however, in another unpublished experimental study, we have assessed the survival of implanted cells after treatment in the MI or MS groups by means of RT-PCR. Myoblasts from male rats were implanted into female recipients, and the hearts were removed 1 day after Ctx. Genomic DNA was purified from the whole heart, and the content of male-specific DNA, which represented the content of implanted cells after treatment, was measured. MS versus MI group results emerged as follows: $3.71 \pm 0.55(P<.05)$ versus $1.71 \pm 0.50$ at day 1 . From these results, we also deduce better myoblast survival with sheet implantation. Although this evaluation only provides a global assessment of cell survival (eg, myoblasts and fibroblasts), we find it compatible with our other findings.

Color kinesis echocardiography results from our past study showed no recovery in regional systolic function after the fibroblast sheet implantation, ${ }^{13}$ and Hutcheson and associates $^{32}$ also showed failure in improvement of systolic function by fibroblast transplantation compared with myoblast transplantation. Mesh implantation only supports the preservation of LV geometry but without improvement in its regional systolic function. ${ }^{33}$ These findings prevented us from using fibroblast sheet or mesh as a control group. We used biodegradable collagen sheets to avoid chest adhesion, and their ineffectiveness on remodeling of myocardial geometry is clearly addressed by control group results.

We conclude that tissue cardiomyoplasty is better than cellular cardiomyoplasty because it repairs the infarcted myocardial wall and recruits supportive HSCs to attenuate cardiac remodeling and improve cardiac performance.

We thank Masako Yokoyama for expert assistance with RTPCR and Shigeru Miyagawa for echocardiography.

\section{References}

1. Quaini F, Kajstura J, Anversa P, et al. Chimerism of the transplanted heart. $N$ Engl J Med. 2002;346:5-15.

2. Nygren JM, Jovinge S, Jacobsen SE, et al. Bone marrow-derived hematopoietic cells generate, cardiomyocytes at a low frequency through cell fusion, but not transdifferentiation. Nat Med. 2004;10: 494-501.

3. Piano MR, Carrigan TM. Cellular cardiomyoplasty: a new therapeutic approach for regenerating the myocardium. J Cardiovasc Nurs. 2003; 18:374-81.

4. Pagani FD, Aretz TH, Aaronson KD, et al. Autologous skeletal myoblasts transplanted to ischemic-damaged myocardium in humans. J Am Coll Cardiol. 2003;41:879-88.

5. Suzuki K, Murtuza B, Yacoub MH, et al. Single fibers of skeletal muscle as a novel graft for cell transplantation to the heart. $J$ Thorac Cardiovasc Surg. 2002;123:984-93.

6. Menasche P, Hagege AA, Duboc D, et al. Autologous skeletal myoblasts transplantation for severe postinfarction left ventricular dysfunction. J Am Coll Cardiol. 2003;1078-83.

7. Taylor DA, Glower DD, Kraus WE, et al. Regenerating functional myocardium; improved performance after skeletal myoblast transplantation. Nat Med. 1998;4:929-33.

8. Okano T, Yamada N, Sakai H, et al. A novel recovery system for cultured cells using plasma-treated polystyrene dishes grafted with poly (N-isopropylacrylamide). J Biomed Mater Res. 1993;27:1243-51.

9. Shaw P, Sheetz M, Kuhlbrandt W. Cell junctions, cell adhesion, and the extracellular matrix. In: Alberts B, Johnson A, Lewis J, Raff M, Roberts K, Walter P. Molecular biology of the cell. New York: Garland Science; 2004. p. 1065-126.

10. Zimmermann WH, Kostin S, Eschenhagen T, et al. Tissue engineering of a differentiated cardiac muscle construct. Circ Res. 2002;90:223-30.

11. Shimizu T, Umezu M, Okano T, et al. Fabrication of pulsatile cardiac tissue grafts using a novel 3-dimensional cell sheet manipulation technique and temperature-responsive cell culture surfaces. Circ Res. 2002;90:e40-8.

12. Okano T, Yamamoto M, Shimizu T, et al. Cell sheet engineering for myocardial tissue reconstruction. Biomaterials. 2003;24:2309-16.

13. Miyagawa S, Memon I, Sawa Y, et al. Efficacy of a novel cellular cardiomyoplasty using cardiac sheet for myocardial regeneration: compared with needle injection. Circulation. 2002;106(19 suppl): II420

14. Askari AT, Unzek S, Penn MS, et al. Effect of stromal-cell-derived factor 1 on stem-cell homing and tissue regeneration in ischemic cardiomyopathy. Lancet. 2003;362:697-703.

15. Ratajczak MZ, Peiper S, Janowska WA, et al. Expression of functional CXCR4 by muscle satellite cells and secretion of SDF-1 by musclederived fibroblasts is associated with the presence of both muscle 
progenitors in bone marrow and hematopoietic stem/progenitor cells in muscles. Stem Cells. 2003;21:363-71.

16. Memon IA, Yoshiki S, Matsuda H, et al. A combined autologous cellular cardiomyoplasty with skeletal myoblasts and bone marrow cells in the canine hearts for ischemic cardiomyopathy. $J$ Thorac Cardiovasc Surg. 2005. In press.

17. Shimizu T, Yamato M, Okano T, et al. Cell sheet engineering for myocardial tissue reconstruction. Biomaterials. 2003;24:2309-16.

18. Weisman HF, Bush DE, Mannisi JA, et al. Cellular mechanism of myocardial infarct expansion. Circulation. 1988;78:186-201.

19. Tori M, Kitagawa-Sakakida S, Shirakura R, et al. Initial T-cell activation required for transplant vasculopathy in retransplanted rat cardiac allografts. Transplantation. 2000;70:737-46.

20. Kitagawa-Sakakida S, Sawa Y, Matsuda H, et al. Selective chemokine and receptor gene expressions in allografts that develop transplant vasculopathy. J Heart Lung Transplant. 2002;21:1090-100.

21. Lanza RP, Langer R, Vacanti J. Principles of tissue engineering. Academic Press; New York: 2003. p. 13-5.

22. Zimmermann WH, Didie M, Eschenhagen T, et al. Cardiac grafting of engineered heart tissue in syngeneic rats. Circulation. 2002;106(suppl I): I151-7.

23. Kofidis T, Akhyari $\mathrm{P}$, Haverich A, et al. In Vitro engineering of heart muscle: artificial myocardial tissue. J Thorac Cardiovasc Surg. 2002; 124:63-9.

24. Kushida A, Yamato M, Okano T, et al. Decrease in culture temperature releases monolayer endothelial cell sheets together with deposited fibronectin matrix from temperature-responsive culture surfaces. J Biomed Mater Res. 1999;45:355-62.
25. Tatsumi R, Anderson JE, Allen RE, et al. HGF/SF is present in normal adult skeletal muscle and is capable of activating satellite cells. Dev Biol. 1998;194:114-28.

26. Balsam LB, Weissman IL, Robbins RC, et al. Hematopoietic stem cells adopt mature hematopoietic fates in ischemic myocardium. $\mathrm{Na}$ ture. 2004;428:668-73.

27. Murry CE, Soonpaa MH, Field LJ, et al. Hematopoietic stem cells do not transdifferentiate into cardiac myocytes in myocardial infarcts. Nature. 2004;428:664-8.

28. Orlic D, Kajstura J, Anversa P. Bone marrow cells regenerate infarcted myocardium. Nature. 2001;410:701-5.

29. Beltrami AP, Kajstura J, Anversa P, et al. Adult cardiac stem cells are multipotent and support myocardial regeneration. Cell. 2003;114:76376.

30. Oh H, Bradfute SB, Schneider MD, et al. Cardiac progenitor cells from adult myocardium: Homing, differentiation, and fusion after infarction. Proc Natl Acad Sci U S A. 2003;100:12313-8.

31. Dreyfus PA, Chretien F, Gherardi RK, et al. Adult bone marrowderived stem cells in muscle connective tissue and satellite cell niches. Am J Pathol. 2004;164:773-9.

32. Hutcheson KA, Atkins BZ, Taylor DA, et al. Comparison of benefits on myocardial performance of cellular cardiomyoplasty with skeletal myoblasts and fibroblasts. Cell Transplant. 2000;9:359-68.

33. Kelley ST, Malekan R, Edmunds LH, et al. Restraining infarct expansion preserves left ventricular geometry and function after acute anteroapical infarction. Circulation. 1999;99:135-42. 